epileptic syndromes and encephalopathies. The phenotype in this small family is a consequence of a homozygous mutation in the TNK2 gene, resulting in a gain of function. (Hitomi Y, Heinzen EL, Donatello S, et al. Ann Neurol 2013 Sep;74(3):496501).

\title{
SUBCLINICAL POSTTRAUMATIC SEIZURES DETECTED BY CONTINUOUS VIDEO-EEG MONITORING
}

Investigators at Mattel Children's Hospital, UCLA, and University of Colorado, used continuous video-EEG monitoring (cEEG) to study the incidence and risk factors for subclinical early posttraumatic seizures (EPTS) in 87 consecutive, unselected (mild severe), acute traumatic brain injury (TBI) patients requiring admission to the PICU. Thirty-seven (42.5\%) had seizures: subclinical in 16.1\% (only subclinical in 6.9\%), status epilepticus (SE) in 18.4\%, and subclinical SE in 13.8\%. Risk factors for subclinical seizures and SE included younger age, abusive head trauma, and intraaxial bleed. SE and subclinical SE were associated with increased hospital length of stay. cEEG monitoring significantly improves detection of seizures and is the only way to detect subclinical seizures (SE). (Arndt DH, Lerner JT, Matsumoto JH, et al. Subclinical early posttraumatic seizures detected by continuous EEG monitoring in a consecutive pediatric cohort. Epilepsia 2013 Oct;54(10):1780-8). (Response: Jason T Lerner, 10833 Le Conte, 22-474 MDCC, Los Angeles, CA 90095. E-mail: jlerner@mednet.ucla.edu).

COMMENT. Continuous EEG monitoring is recommended in young children with TBI, particularly in those with abusive head trauma and in those with intraaxial blood on CT. Rapid detection and treatment of EPTS may be of benefit in the immediate management of patients with TBI, but control of subclinical EPTS may not prevent occurrence of late posttraumatic epilepsy nor reflect long-term adverse effects of AEDs on the developing brain.

\section{PROGNOSIS OF EPILEPSY}

Investigators from the Institute of Neurology, Queen Square, London, UK, report results of longitudinal cohort studies of prognosis in epilepsy in adults and children and focus particularly on the National General Practice Study of Epilepsy (NGPSE) in 1195 patients initiated in 1983. Other longitudinal studies include the Mayo Clinic Record Linkage Study, the Tonbridge Study and the Study from Turku, confined to children and initiated in the 1970s. The findings are summarized as follows: 1) Epilepsy prognosis is frequently good, $65-85 \%$ cases entering long-term remission; 2) prognosis is better in newly diagnosed cases than in patients with chronic epilepsy; 3) early response to treatment is usually an indication of a good long-term prognosis; 4) the longer the remission, the less likely a subsequent recurrence; 5) the longer seizures recur, the poorer the long-term outlook; 6) delaying treatment, even for many years, does not worsen longterm prognosis; 7) continuous and burst patterns are more common than intermittent seizure patterns; 8) mortality may occur at any time in the course of epilepsy but is highest in the early years after diagnosis and is largely due to the underlying cause; 9) febrile seizure prognosis is generally good with $\sim 6-7 \%$ developing late epilepsy (rate of 
epilepsy developing is higher in those with FS occurring before age 1 year or after age 3 years, and with complex FS); and 10) clinical factors associated with a poor prognosis include a neurodeficit, poor response to initial therapy, and some epilepsy syndromes. (Shorvon SD, Goodridge DMG. Longitudinal cohort studies of the prognosis of epilepsy: contribution of the National General Practice Study of Epilepsy and other studies. Brain 2013 Nov;136(Pt 11):3497-510). (Response: Professor Simon Shorvon, E-mail: S.shorvon@ucl.ac.uk).

COMMENT. Ethics committee approval had been obtained at the beginning of the study, which did not require patient consent for collection of anonymized prognostic data. In 2007 a government appointed committee ruled that, with some exceptions, the patient's consent was required for collection of personal data from the general practitioner. The reduction in available data that followed has resulted in the premature termination of the study. The authors comment that this ill-advised decision by government appointed committees has harmed large-scale epidemiological studies in Britain, and specifically resulted in loss of 30-year follow-up of a large cohort of patients with epilepsy and reasons for a persistently high mortality.

\section{INTRACRANIAL TUMORS}

\section{DIAGNOSIS OF INTRACRANIAL GERM CELL TUMORS}

Investigators from the Massachusetts General Hospital, Boston, studied the manifestations and time to diagnosis of 70 children with germ cell tumors (GCTs) treated between 1998 and 2012. The median duration of symptoms before diagnostic MRI was 6 months (range, 2 days to 72 months). Diagnosis was delayed (>6 months) in 38 (54\%). The delay increased the risk of disseminated disease. Thirty patients (43\%) had nongerminomatous tumors (NGGCTs) and 40 (57\%) were diagnosed with pure germinomas (PGs). The majority of primary tumors were located in the suprasellar region (28\% of NGGCT and $40 \%$ of PG) followed by the pineal region (23\% of NGGCT and $33 \%$ of PG). All isolated pineal region tumors occurred in male patients; suprasellar tumors occurred in females in $61 \%$.

Symptoms of GCT were headache (69\%), nausea and vomiting (50\%), polyuria and/or polydipsia in $59 \%$, double vision (34\%), visual field cuts or impaired visual acuity in $27 \%$, poor growth (17\%), and premature puberty in $14 \%$. Pineal tumors presented with symptoms of hydrocephalus, whereas suprasellar tumors caused endocrinopathies. Ophthalmic symptoms occurred in all patients: pineal located tumors caused diplopia and Parinaud syndrome symptoms in $61 \%$, and suprasellar tumors caused visual acuity and/or visual field limitations in $29 \%$. Patients with GCT were evaluated by a broad spectrum of pediatric specialists, and patients with delayed diagnosis were seen by 2 or more physicians and subspecialists: a neurologist in $17 \%$, ophthalmologist $27 \%$, or endocrinologist $34 \%$. An endocrinopathy, especially diabetes insipidus (in 50\%), was diagnosed before the diagnosis of brain tumor. Progressive enlargement of the infundibulum led to biopsy, and diagnosis was confirmed by abnormal levels of human chorionic gonadotropin in the CSF and elevated serum alpha-fetoprotein. (Sethi RV, Marino R, Niemierko A, Tarbell NJ, Yock TI, MacDonald SM. Delayed diagnosis in 